\title{
Positive selection of skeleton-related genes during duck domestication revealed by whole genome sequencing
}

Tao Zhu' ${ }^{1}$ Xin Qi ${ }^{1}$, Yu Chen², Liang Wang ${ }^{2}$, Xueze Lv², Weifang Yang ${ }^{2}$, Jianwei Zhang ${ }^{2}$, Kaiyang Li ${ }^{2}$, Zhonghua Ning ${ }^{1}$, Zhihua Jiang ${ }^{3}$ and Lujiang Qu ${ }^{1 *}$

\begin{abstract}
Background: Domestication alters several phenotypic, neurological, and physiological traits in domestic animals compared to those in their wild ancestors. Domestic ducks originated from mallards, and some studies have shown that spot-billed ducks may have also made minor genetic contributions to domestication. Compared with the two ancestral species, domestic ducks generally differ in body size and bone morphology. In this study, we performed both genomic and transcriptomic analyses to identify candidate genes for elucidating the genetic mechanisms underlying phenotypic variation.
\end{abstract}

Methods: In this study, the duck genome data from eight domestic breeds and two wild species were collected to study the genetic changes during domestication. And the transcriptome data of different tissues from wild ducks and seven domestic ducks were used to reveal the expression difference between wild and domestic ducks.

Results: Using fixation index (Fst) algorithm and transcriptome data, we found that the genes related to skeletal development had high Fst values in wild and domestic breeds, and the differentially expressed genes were mainly enriched in the ossification pathway. Our data strongly suggest that the skeletal systems of domestic ducks were changed to adapt to artificial selection for larger sizes. In addition, by combining the genome and transcriptome data, we found that some Fst candidate genes exhibited different expression patterns, and these genes were found to be involved in digestive, immune, and metabolic functions.

Conclusions: A wide range of phenotypic differences exists between domestic and wild ducks. Through both genome and transcriptome analyses, we found that genes related to the skeletal system in domestic ducks were strongly selected. Our findings provide new insight into duck domestication and selection effects during the domestication.

Keywords: Duck, Domestication, Artificial selection, Positive selection, Skeleton

*Correspondence: quluj@163.com

1 Department of Animal Genetics and Breeding, National Engineering Laboratory for Animal Breeding, College of Animal Science

and Technology, China Agricultural University, Yuanmingyuan West Road 2\#, Beijing 100193, China

Full list of author information is available at the end of the article

\section{Background}

Domestication is the process in which a wild species is artificially selected to be domestic, with many traits changed to meet human requirements. Various features distinguish domestic animals from their wild ancestors, and it is well-documented that the genetic architecture underlying variations in phenotypic traits (such as morphology in chickens $[1,2]$, pigs [3-5], and pigeons [6, 7]; 
feather color $[8,9]$; neurological aspects such as tameness in companion animals [10-12]; and physiological aspects such as starch digestibility in dogs [13] and reproduction in chickens $[14,15])$ are altered dramatically in domestic animals compared to those in their wild ancestors.

Archeological evidence shows that China has a long history in ducks domestication, the origin of domestic ducks is controversial. A previous study showed that Chinese domestic ducks mainly originated from mallard (Anas platyrhynchos) and may have been introgressed by spot-billed ducks (Anas zonorhyncha) [16]. Compared to wild ducks, domestic ducks do not require evasion from predators and foraging, causing domestic ducks to retain their various plumage colors [9], such as white in Pekin ducks. Furthermore, wild ducks maintain their flight capability for migration, whereas domestic ducks are flightless. The domestication process has greatly changed the body shape, including the size, elevation, and fat content. These phenotypic differences indicate that large changes have occurred in the genome, and is important for animal genetics to identify the genes selected during the domestication process.

In this study, two wild duck species and eight domestic duck breeds were selected to investigate the potential selected genes in domesticated ducks using genomic and transcriptomic data. We found that some genes associated with bone development were positively selected and differed at both the genomic and transcriptomic levels between wild and domestic ducks.

\section{Methods}

\section{Sampling and genomic and transcriptomic sequencing}

The genome sequencing data of two ancestral wild populations and eight domestic breeds were used in this study. The ancestral wild duck including mallard $(\mathrm{MD}, \mathrm{n}=21)$ and spot-billed ducks (SB), domesticated populations including Pekin duck (PK, $\mathrm{n}=8$ ), Cherry Valley duck $(\mathrm{CV}, \mathrm{n}=8)$, Maple Leaf duck $(\mathrm{ML}, \mathrm{n}=8)$, Jinding duck (JD, $\mathrm{n}=8$ ), Shaoxing duck (SX, $\mathrm{n}=8$ ), Mei duck (Mei, $\mathrm{n}=8$ ), Shanma duck (SM, $\mathrm{n}=8$ ), and Gaoyou duck (GY, $\mathrm{n}=8$ ). Mei was obtained from Anhui Province, China, SB was collected from Ningxia Province, China. The remaining ducks were obtained from our previous study [8]. Blood samples were collected from the brachial veins by standard venipuncture, and all the ducks were released. Genomic DNA was extracted from newly collected samples using the standard phenol/chloroform extraction method, and two paired-end libraries (500 bp) were constructed using the Illumina HiSeq 2500 sequencing platform (San Diego, CA, USA). In addition, the liver, brain, and breast muscle tissues from 14 adult male ducks (MD, $\mathrm{n}=7 ; \mathrm{PK}, \mathrm{n}=1 ; \mathrm{CV}, \mathrm{n}=1 ; \mathrm{ML}, \mathrm{n}=1 ; \mathrm{JD}, \mathrm{n}=1$; $\mathrm{SM}, \mathrm{n}=1 ; \mathrm{SX}, \mathrm{n}=1 ; \mathrm{GY}, \mathrm{n}=1$ ) were collected for RNA sequencing (RNA-seq). The detailed sample information and sequencing methods have been described in our previous study [8].

\section{Pretreatment of genomic data}

The raw genome data were first processed using fastp (v0.20.0) to filter adapter contamination and low-quantity reads [17]. The clean data were aligned to the duck genome (https://www.ncbi.nlm.nih.gov/, accession: GCF_003850225.1) using BWA-MEM (v0.7.15) [18]. Picard (http://broadinstitute.github.io/picard/) was used to sort the aligned data and remove duplicate reads. Genome Analysis Toolkit v3.6 (https://gatk.broadinsti tute.org/) was used for variant calling and variant filtering. SNPs were filtered using the following rules: (a) QUAL > 30.0, (b) QD $>5.0$, (c) FS $<60.0$, (d) $\mathrm{MQ}>40.0$, (e) MQRankSum $>-12.5$, and (f) ReadPosRankSum $>-8.0$. Additionally, if there were more than three SNPs clustered in a 10-bp window, they were all considered as false positives and removed [19]. Finally, credible SNPs in VCF format were retained for subsequent analysis.

\section{Population structure and phylogenetic trees}

The SNP file was converted to plink format using VCFtools (v0.1.13) [20], and low-quality SNPs with a missing rate greater than 0.1 [21]. Principal component analysis was conducted with plink [22], and the top 20 components were used for population relationship analysis. To construct the phylogenetic tree, SNPs that overlapped with the CDS were extracted, and the maximum likelihood phylogenetic tree was constructed using iqtree 2 with the GTR + ASC model [23]. iTOl was used to visualize the phylogenetic trees [24].

\section{Selective-sweep analysis}

To define candidate direct selection regions, the wild ducks (MD and $\mathrm{SB}$ ) were placed in one group, and the remaining ducks were clustered into another group. The Fst was calculated with $50-\mathrm{kb}$ windows and slides with 25-kb steps; genome regions overlapping with upstream and downstream $50 \mathrm{~kb}$ of the top 1\% Fst windows were considered as candidate regions, and genes with overlapping candidate regions were considered as candidate genes.

\section{RNA-seq data analysis}

Raw transcriptome data were processed using fastp (v0.20.0) [17], and the clean data were aligned to the duck genome (https://www.ncbi.nlm.nih.gov/, accession: GCF_003850225.1) using HISAT2 (v 2.1.0) [25]. The aligned files were sorted using SAMtools (v 1.9) [26]. The reads number for each gene was counted using featureCounts (1.6.4) [27]. The reads count files were merged 
with tissue, and expression levels were calculated using DESeq2 [28]. Genes with an absolute value of expression level change greater than 1.5 -fold and adjusted $p$ value less than 0.05 were considered as DEGs. Metascape was used for gene enrichment analysis [17].

\section{Results}

\section{Population structure}

From the genome data, we obtained 38,097,848 highquality single-nucleotide polymorphisms (SNPs) with an average depth of 564 . To study the population structure, we calculated the top 20 components of the experimental ducks; the top three components explained $37.68 \%$ of the total variance (Fig. 1A). The results showed that the wild and domesticated ducks were classified by a line in which component 1 equals 0 , whereas component 1 of wild ducks was less than 0 (Fig. 1A). All individuals were clustered into three groups, with the wild species MD and SB separated from PK, CV, and ML ducks, which were clustered into one group, and the remaining ducks clustered together in another group. To study the phylogenetic relationships among the ducks, coding sequence (CDS) SNPs were used to construct the maximum likelihood evolutionary tree, which showed that $\mathrm{SB}$ and $\mathrm{MD}$ were clustered into one branch and $\mathrm{PK}, \mathrm{CV}$, and ML were clustered into another branch, and the remaining breeds were grouped together (Fig. 1B).

\section{Skeletal system development genes were positively} selected during domestication

The slide window fixation index (Fst) algorithm was employed to identify candidate genes that diverged between wild and domesticated ducks. To avoid bias caused by sex chromosomes, only autosomes were further analyzed. We obtained 39,251 autosome windows, with a mean Fst of 0.061 between wild and domesticated ducks. The two peaks of chromosomes 16 and 8 exceeded 0.2. The maximum Fst value was 0.22 at chromosome $16: 300,001 \mathrm{bp}-350,001 \mathrm{bp}$, which overlapped with the TMEM132B gene, and the second peak at chromosome 8:19,750,001 bp-19,800,001 bp, overlapping with the LOC101797367 gene, also known as CLAC4 (Fig. 2). We extracted genes overlapping with the top $1 \%$ window or adjacent windows as candidate genes. Finally, we identified 525 candidate genes and 260 genes mapped to the human genome. Metascape was used to annotate the gene functions [29]. Many gene ontology (GO) items were related to metabolism and cellular energy conversion (Fig. 3A). One GO item, GO:0001501, was significantly correlated with skeletal system development, and 14 genes were included in this item. Enrichment analysis on the DisGeNET platform showed that the candidate genes were related to bone diseases, such as osteosclerosis, bone pain, and abnormality of the metaphyses (Fig. 3B) [30]. In addition, the EIF2AK3 gene on chromosome 4 overlapped with the third highest peak.
A

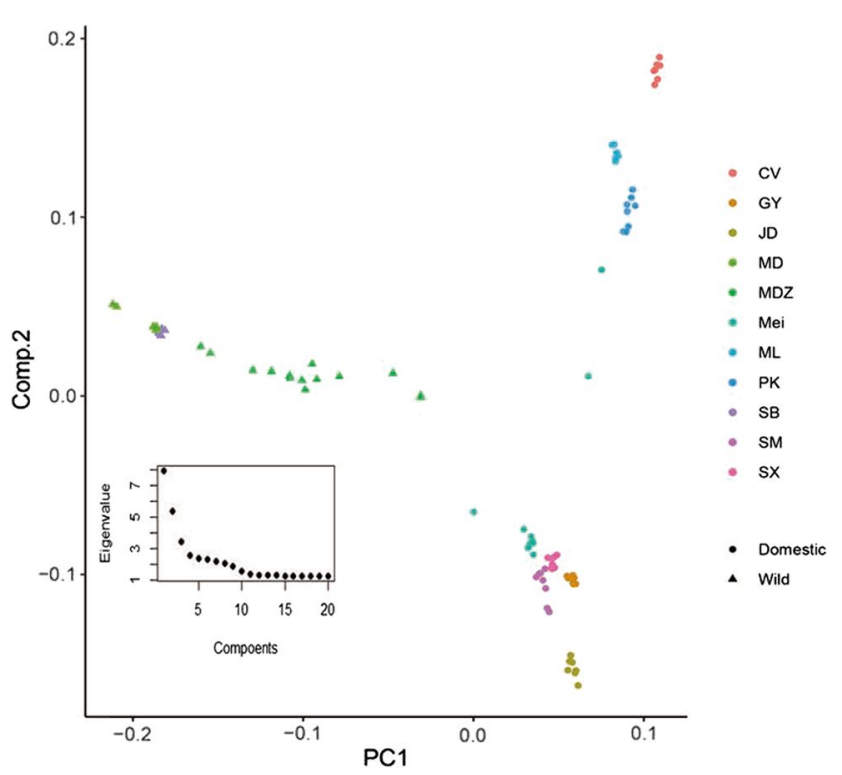

B

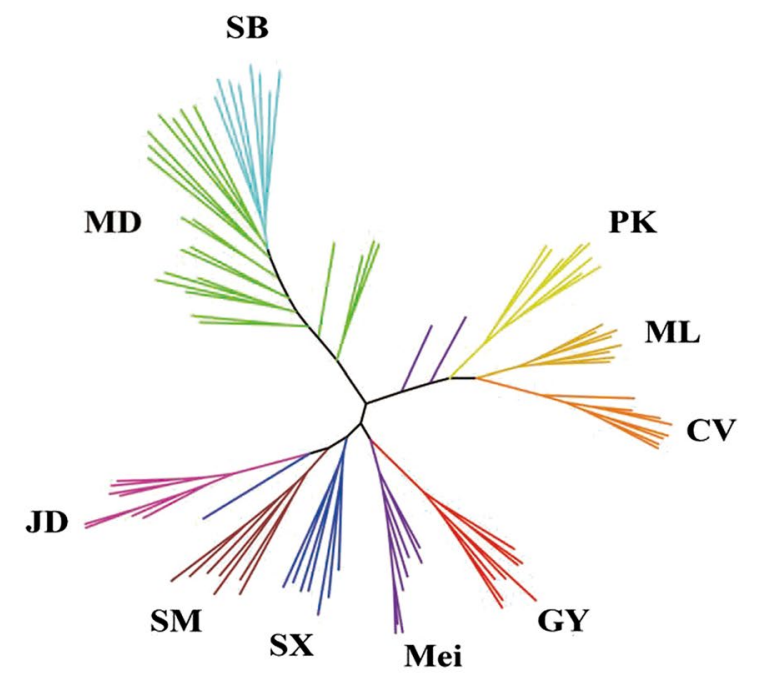

Fig. 1 Population structure of ducks. A Principal component analysis plot of ducks. The inner dot plot represents the eigenvalues of the top 20 principal components. B Maximum likelihood phylogenetic tree of all ducks 


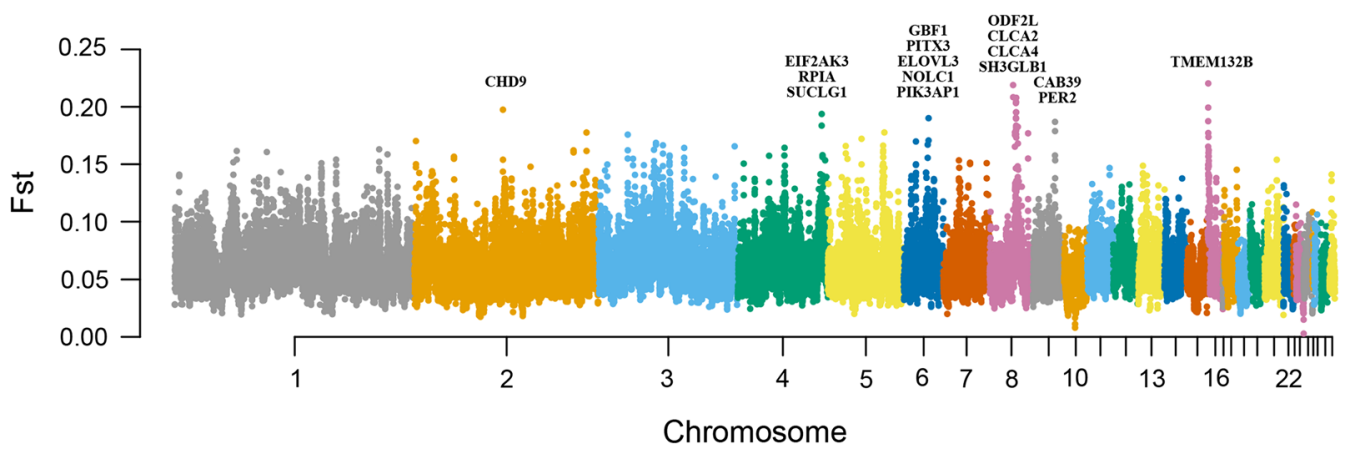

Fig. 2 Manhattan plot for Fst and genes that overlap with sweep peaks

This gene was associated with body size, abnormal skeletal morphology, and abnormal hind limb morphology in mice $[31,32]$.

\section{Differentially expressed genes (DEGs) in muscle predominantly enrichedin bone developmental pathway} Gene function can be determined based on the RNA transcription level at a specific time. The breast muscle, liver, and brain from wild and domesticated male ducks were collected for gene expression analysis. The expression level of each gene was standardized using the FPKM algorithm [33], and DEGs were identified by their foldchange and adjusted $p$ value. After filtration, 112, 31, and 180 genes in the liver, brain, and breast muscle, respectively, were identified as DEGs. The number of DEGs in the breast muscle was significantly larger than that in the brain and liver. GO analysis was used for gene function analysis. DEGs in the breast muscle were enriched in the ossification and bone development pathways (Fig. 3C). DEGs in the liver were enriched in the muscle organ development and cartilage development pathways (Additional file 1: Figure S1A). The brain was enriched in the visual perception and hormone regulation pathways (Additional file 1: Figure S1B).

Next, we extracted the overlapping genes between the Fst and DEGs analyses, and identified 6, 2, and 10 genes in the liver, brain, and breast muscle, respectively, showing different expression patterns and high Fst values (Table 1). These genes are involved in the immune, digestive, and metabolic systems. The most divergent gene, TMEM132B, was highly expressed in the wild duck breast muscle.

\section{Discussion}

Domestication, including captivity and artificial selection, has resulted in large morphological changes in ducks. Breeding has also introduced a large number of variants into the domesticated duck genome. In our study, wild and domesticated ducks were distinguished by the first principal component (Fig. 1A), which showed that long-term artificial selection led to tremendous variation in the duck genomes. In previous studies, the differences were mainly reflected in agronomic traits, such as egg production, growth rate, and fat deposition [8,34]. Domestic ducks were clustered into two groups by the second principal component, corresponding to their biological origin. Our results suggest that genetic distances in Chinese indigenous ducks are related to geographical distances, and indigenous ducks in southern China may have originated from a common ancestor. With the development of sequencing technology, phylogenetic analyses of ducks have been performed at the wholegenome scale. These studies demonstrated that mallards are the ancestors of domestic ducks [8,9], and Liu et al. [35] found that MD and SB specific SNPs were equally distributed into eight domestic ducks. Taken together, both MD and SB ducks are thought to have contributed to domestic ducks.

In our study, the Fst algorithm was used to identify divergent genes. In total, 525 genes were identified as candidate divergent genes. One GO item containing 14 genes was related to bone development, and the DisGeNET database showed that our candidate genes were related to bone diseases. Furthermore, bone-related DEGs in the breast muscle showed different expression patterns, suggesting that the musculoskeletal system changed dramatically during duck domestication. These changes may be attributed to the limited activity space during domestication. In chickens, bone mineral density, trabecular bone volume, and trabecular microarchitecture were found to be significantly reduced when chickens were kept in small cages [36]. Therefore, domestic ducks may have lower bone strength and density. In our study, we found that the EIF2AK3 gene greatly differed between domestic and wild ducks, and many studies showed that mutations in the EIF2AK3 gene can lead 


\section{A}

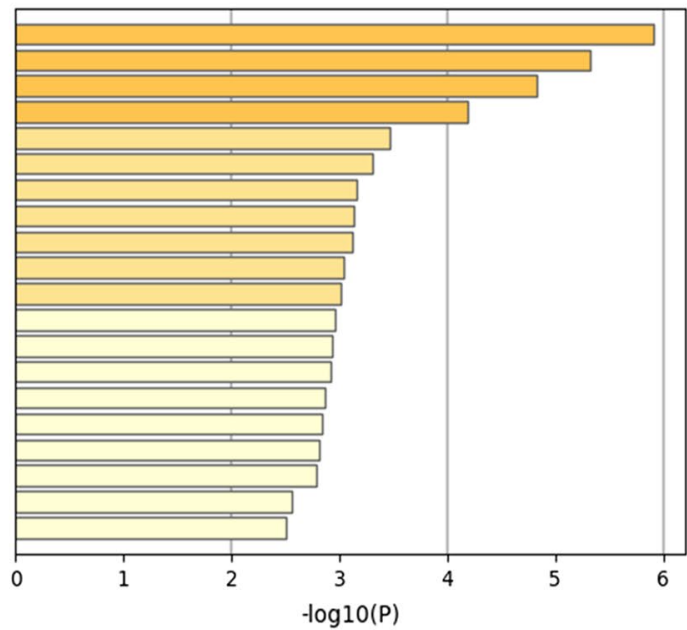

R-HSA-1500931: Cell-Cell communication CORUM:2850: ITGA5-ITGB1-FN-1-NOV complex R-HSA-8874081: MET activates PTK2 signaling GO:0036507: protein demannosylation

R-HSA-196849: Metabolism of water-soluble vitamins and cofactors GO:0042559: pteridine-containing compound biosynthetic process GO:0071347: cellular response to interleukin-1

GO:0098742: cell-cell adhesion via plasma-membrane adhesion molecules

GO:0030029: actin filament-based process

R-HSA-8953854: Metabolism of RNA

GO:0006403: RNA localization

GO:0090278: negative regulation of peptide hormone secretion

GO:0045056: transcytosis

R-HSA-194315: Signaling by Rho GTPases

GO:0099515: actin filament-based transport

GO:0006855: drug transmembrane transport

GO:0032402: melanosome transport

GO:0001501: skeletal system development

GO:0006631: fatty acid metabolic process

R-HSA-9660821: ADORA2B mediated anti-inflammatory cytokines production

B

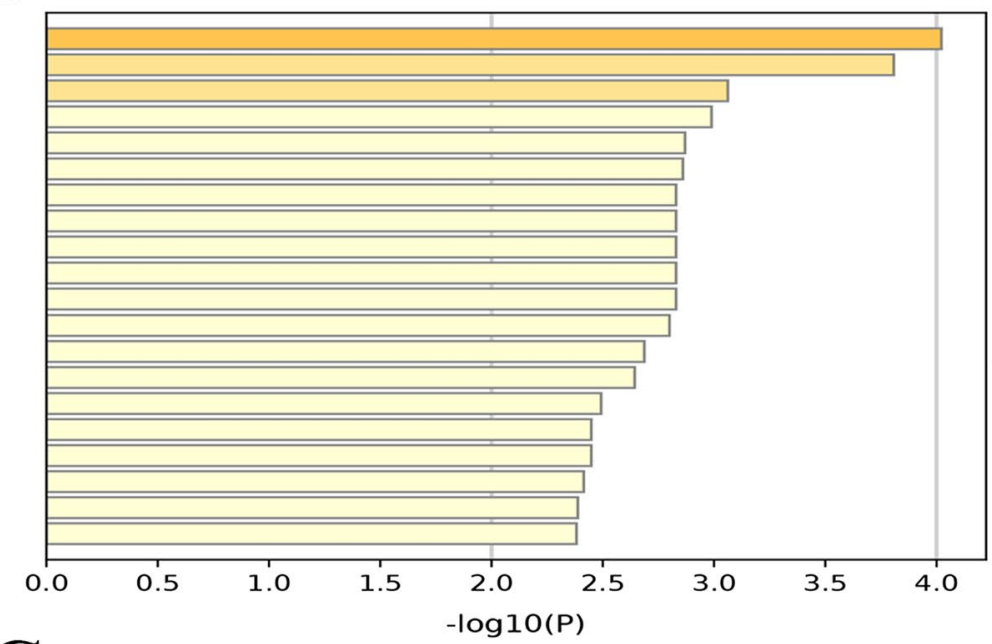

Osteosclerosis

Abnormality of the metaphyses

Hypophosphatemia

Bone pain

Tremor

Short thorax

Class III malocclusion

Hypertrophy of lower jaw

mandibular excess (physical finding)

Increased size of mandible

Mandibular hyperplasia

Delayed myelination

Joint stiffness

Short stature

Choreatic disease

Chorea

Alkaline phosphatase serum increased

Abnormal skeletal development

Renal Cell Carcinoma

Infantile onset

C

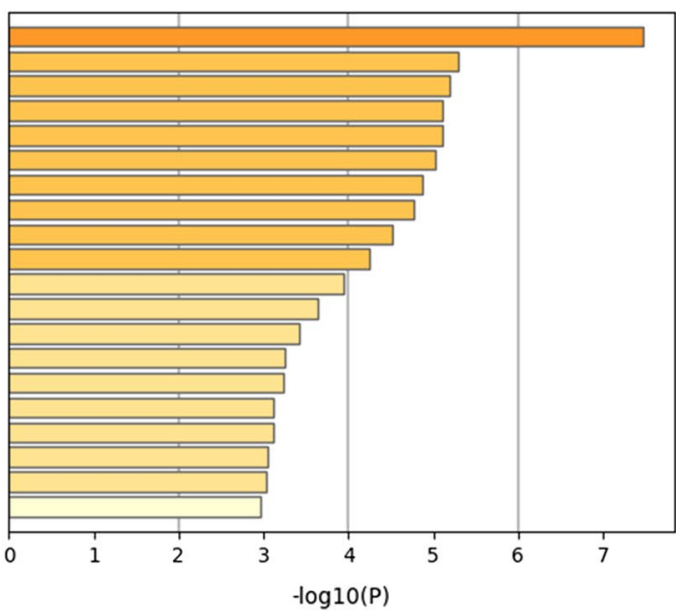

GO:0001503: ossification

GO:1905517: macrophage migration

GO:0009074: aromatic amino acid family catabolic process

GO:0010720: positive regulation of cell development

GO:1903522: regulation of blood circulation

GO:0035973: aggrephagy

GO:0043269: regulation of ion transport

GO:0001501: skeletal system development

GO:0006935: chemotaxis

GO:0007507: heart development

GO:0007169: transmembrane receptor protein tyrosine kinase signaling pathway

hsa 04080: Neuroactive ligand-receptor interaction

GO:0051051: negative regulation of transport

GO:0006970: response to osmotic stress

GO:0051250: negative regulation of lymphocyte activation

GO:0048608: reproductive structure development

GO:0001764: neuron migration

GO:0040008: regulation of growth

GO:0043062: extracellular structure organization

GO:0001823: mesonephros development

$-\log 10(P)$

Fig. 3 Gene enrichment analysis for candidate genes. A Gene Ontology (GO) analysis for Fst candidate genes. B DisGeNET enrichment result for Fst candidate genes. C GO analysis for DEG in the breast muscle 
Table 1 Differentially expressed candidate genes in the breast, liver, and brain

\begin{tabular}{lll}
\hline Breast & Liver & Brain \\
\hline HHLA2 (immune) & LOC113842605 & LOC113844317 \\
IFT57 (body development) & LOC106019145 & LOC106015673 \\
LOC101795816 (immune) & NMNAT2 (metabolism) & \\
LOC106019145 & LAMC2 (body develop- & \\
ment) & \\
LOC101798418 (metabo- & LOC101794647 (metabo- & \\
lism) & lism) \\
SGMS2 (bone) & LOC113844272 (metabo- \\
DDX60 (immune) & lism) \\
LOC101800182 (metabo- & \\
lism) & \\
LOC110351937 & \\
LOC106017289 & \\
\hline
\end{tabular}

Terms in parentheses represent the gene functional classification

to Wolcott-Rallison syndrome [37-39], a rare autosomal recessive disorder characterized by permanent insulindependent diabetes, multiple epiphyseal dysplasia, and growth retardation. In mice, EIF2AK3 is associated with the hindlimb morphology and body weight $[31,32,40]$. The EIF2AK3 gene plays an important role in the development and morphology of the domestic duck bone. This functional change in bone development may have led to the loss of flight in domestic ducks. Compared to their wild relatives, wild birds have a lighter mass, larger wing area, and greater bone length [41-43]. These differences are consistent with the major breeding goals. For example, as one of the most famous meat-type ducks worldwide, PK were bred for their fast growth rate and high fat deposition; additionally, their body weight can reach up to $3.1 \mathrm{~kg}$ at 35 days of age, whereas the adult MD weighs only $1.1-1.2 \mathrm{~kg}$ [44-47]. Although breeding greatly increased the body weight of ducks, long-term captivity weakened the importance of wings, which was accompanied by physiological changes such as flight muscle atrophy and decreased bone strength and wing size (limbs). For example, in a flightless steam duck (Tachyeres patachonicus), wing loading, humerus length, radius length, and ulna length were significantly changed compared to those in their flying relatives [41].

TMEM132B and CLCA4 showed the highest Fst values. CLCA4 is involved in regulating electrolytic fluxes and thereby modulates secretion, absorption, cell volume, and membrane potential. CLCA4 was also found to be highly expressed in the human colon and esophagus, and variants of CLCA4 were associated with cystic fibrosis $[48,49]$, an inherited disorder that severely damages the lungs and digestive system. Selection of the CLCA4 gene may be related to changes in food composition. The TMEM132B is a member of the TMEM132 gene family [50]. The TMEM132 family is composed of neuronal genes, and mutations in the TMEM132 gene can cause nervous system diseases such as insomnia symptoms and hearing loss [51, 52]. A similar study in cattle showed that TMEM132E was positively selected [53]. Transcriptomic analysis revealed that some Fst candidate genes, mainly those with immune and metabolic functions, had different expression patterns. Interestingly, two genes (LOC101794647 and LOC113844272, also known as pancreatic alpha-amylase and pancreatic alpha-amylase-like, respectively) were found to be related to starch digestion, possibly because of the grain present in domestic duck feed. A similar selection effect was found in dogs, in which the starch digestion gene MGAM was selected and highly expressed [13]. In addition to bone development, our data suggest that the digestive, nervous, and immune systems were impacted during selection for breeding.

\section{Conclusions}

By comparing two wild duck species with domesticated duck breeds using both genomic and transcriptomic data, we found that domestic ducks changed in many aspects during domestication, particularly in the skeletal system.

\section{Abbreviations}

MD: Mallard; SB: Spot-billed duck; PK: Pekin duck; CV: Cherry Valley duck; ML: Maple Leaf duck; JD: Jinding duck; SX: Shaoxing duck; Mei: Mei duck; SM: Shanma duck; GY: Gaoyou duck; SNPs: Single-nucleotide polymorphisms; Fst: Fixation index; DEGs: Differentially expressed genes; CDS: Coding sequence.

\section{Supplementary Information}

The online version contains supplementary material available at https://doi. org/10.1186/s12862-021-01894-7.

Additional file 1: Figure S1. Gene Ontology (GO) analysis of differentially expressed genes in the liver $(A)$ and brain (B)

\section{Acknowledgements}

Not applicable.

Authors' contributions

LQ conceived the study and participated in its design, TZ and XQ analyzed the data, TZ wrote the manuscript, ZN and ZJ made substantive revisions to the manuscript, and $Y C, L W, X L, W Y, K L$, and JZ contributed to sample collection. All authors read and approved the final manuscript.

\section{Funding}

This work was supported by the Beijing Innovation Consortium of Agriculture Research System (BAIC04-2020). The funding bodies played no role in the design of the study, collection, analysis, and interpretation of data, or in writing the manuscript.

\section{Availability of data and materials}

The data supporting the conclusions of this article are available from NCBI (https://www.ncbi.nlm.nih.gov/, PRJNA686828). 


\section{Declarations}

\section{Ethics approval and consent to participate}

No animals were killed for this study. Sample collection was performed using strict protocols approved by the Animal Welfare Committee of the China Agricultural University (permit XK622). The spot-billed ducks and Mei ducks was sampled with permission from Institute of Animal Science of Chinese Academy of Agricultural Sciences (Permit 046/2018).

\section{Consent for publication}

Not applicable.

\section{Competing interests}

The authors declare that they have no competing interests.

\section{Author details}

'Department of Animal Genetics and Breeding, National Engineering Laboratory for Animal Breeding, College of Animal Science and Technology, China Agricultural University, Yuanmingyuan West Road 2\#, Beijing 100193, China. ${ }^{2}$ Beijing General Station of Animal Husbandry, Beiyuan Road 15A\#, Beijing 100107, China. ${ }^{3}$ Department of Animal Sciences, Center for Reproductive Biology, Veterinary and Biomedical Research Building, Washington State University, Pullman, Washington 647010, USA

Received: 28 December 2020 Accepted: 20 August 2021

Published online: 06 September 2021

\section{References}

1. Stange M, Núñez-León D, Sánchez-Villagra M, Jensen P, Wilson L. Morphological variation under domestication: how variable are chickens? R Society Open Sci. 2018;5:180993.

2. Duggan BM, Hocking PM, Schwarz T, Clements DN. Differences in hindlimb morphology of ducks and chickens: effects of domestication and selection. Genet Selection Evol. 2015;47(1):88.

3. Frantz LAF, Schraiber JG, Madsen O, Megens H-J, Cagan A, Bosse M, Paudel Y, Crooijmans RPMA, Larson G, Groenen MAM. Evidence of long-term gene flow and selection during domestication from analyses of Eurasian wild and domestic pig genomes. Nat Genet. 2015;47(10):1141-8.

4. Geiger M, Sánchez-Villagra MR. Similar rates of morphological evolution in domesticated and wild pigs and dogs. Front Zool. 2018;15(1):23.

5. Neaux D, Sansalone G, Lecompte F, Noûs C, Haruda A, Schafberg R, Cucchi T. Examining the effect of feralization on craniomandibular morphology in pigs, Sus scrofa (Artiodactyla: Suidae). Biol J Lin Soc. 2020;131(4):870-9.

6. Bartels T. Variations in the morphology, distribution, and arrangement of feathers in domesticated birds. J Exp Zool Part B Mol Dev Evol. 2003;298B(1):91-108.

7. Stringham Sydney A, Mulroy Elisabeth E, Xing J, Record D, Guernsey Michael W, Aldenhoven Jaclyn T, Osborne Edward J, Shapiro MD. Divergence, convergence, and the ancestry of feral populations in the domestic rock pigeon. Curr Biol. 2012;22(4):302-8.

8. Zhang Z, Jia Y, Almeida P, Mank JE, van Tuinen M, Wang Q, Jiang Z, Chen $Y$, Zhan $\mathrm{K}$, Hou S, et al. Whole-genome resequencing reveals signatures of selection and timing of duck domestication. GigaScience. 2018;7(4):giy027.

9. Zhou Z, Li M, Cheng H, Fan W, Yuan Z, Gao Q, Xu Y, Guo Z, Zhang Y, Hu J, et al. An intercross population study reveals genes associated with body size and plumage color in ducks. Nat Commun. 2018;9(1):2648.

10. Brusini I, Carneiro M, Wang C, Rubin C-J, Ring H, Afonso S, Blanco-Aguiar $J A$, Ferrand N, Rafati N, Villafuerte R, et al. Changes in brain architecture are consistent with altered fear processing in domestic rabbits. ProcNatl Acad Sci. 2018;115(28):7380

11. Huang S, Slomianka L, Farmer AJ, Kharlamova AV, Gulevich RG, Herbeck YE, Trut LN, Wolfer DP, Amrein I. Selection for tameness, a key behavioral trait of domestication, increases adult hippocampal neurogenesis in foxes. Hippocampus. 2015;25(8):963-75.

12. Sato DX, Rafati N, Ring H, Younis S, Feng C, Blanco-Aguiar JA, Rubin C-J, Villafuerte R, Hallböö F, Carneiro M, et al. Brain transcriptomics of wild and domestic rabbits suggests that changes in dopamine signaling and ciliary function contributed to evolution of tameness. Genome Biol Evol. 2020:12(10):1918-28.

13. Axelsson E, Ratnakumar A, Arendt M-L, Maqbool K, Webster MT, Perloski $\mathrm{M}$, Liberg O, Arnemo JM, Hedhammar A, Lindblad-Toh K. The genomic signature of dog domestication reveals adaptation to a starch-rich diet. Nature. 2013;495(7441):360-4.

14. Ericsson M, Fallahsharoudi A, Bergquist J, Kushnir MM, Jensen P. Domestication effects on behavioural and hormonal responses to acute stress in chickens. Physiol Behav. 2014;133:161-9.

15. Johnsson M, Gustafson I, Rubin C-J, Sahlqvist A-S, Jonsson KB, Kerje S, Ekwall $O$, Kämpe $O$, Andersson $L$, Jensen $P$, et al. A sexual ornament in chickens is affected by pleiotropic alleles at $\mathrm{HAO} 1$ and BMP2, selected during domestication. PLOS Genetics. 2012;8(8):e1002914.

16. Li H-F, Zhu W-Q, Song W-T, Shu J-T, Han W, Chen K-W. Origin and genetic diversity of Chinese domestic ducks. Mol Phylogenet Evol. 2010;57(2):634-40.

17. Chen S, Zhou Y, Chen Y, Gu J. fastp: an ultra-fast all-in-one FASTQ preprocessor. Bioinformatics. 2018;34(17)::884-90.

18. Li H, Durbin R. Fast and accurate short read alignment with BurrowsWheeler transform. Bioinformatics. 2009;25(14):1754-60.

19. Li H, Ruan J. Mapping short DNA sequencing reads and calling variants using mapping quality scores. Genome Res. 2008;18:1851-8.

20. Danecek P, Auton A, Abecasis G, Albers CA, Banks E, DePristo MA, Handsaker RE, Lunter G, Marth GT, Sherry ST, et al. The variant call format and VCFtools. Bioinformatics. 2011;27(15):2156-8.

21. Coop G, Pickrell JK, Novembre J, Kudaravalli S, Li J, Absher D, Myers RM, Cavalli-Sforza LL, Feldman MW, Pritchard JK. The role of geography in human adaptation. PLOS Genetics. 2009;5(6):e1000500.

22. Purcell S, Neale B, Todd-Brown K, Thomas L, Ferreira MAR, Bender D, Maller J, Sklar P, de Bakker PIW, Daly MJ, et al. PLINK: a tool set for whole-genome association and population-based linkage analyses. Am J Hum Genetics. 2007:81(3):559-75.

23. Nguyen L-T, Schmidt HA, von Haeseler A, Minh BQ. IQ-TREE: a fast and effective stochastic algorithm for estimating maximum-likelihood phylogenies. Mol Biol Evol. 2015;32(1):268-74.

24. Letunic I, Bork P. Interactive Tree Of Life (iTOL) v4: recent updates and new developments. Nucleic Acids Res. 2019:47(W1):W256-9.

25. Kim D, Paggi JM, Park C, Bennett C, Salzberg SL. Graph-based genome alignment and genotyping with HISAT2 and HISAT-genotype. Nat Biotechnol. 2019;37(8):907-15.

26. Li H, Handsaker B, Wysoker A, Fennell T, Ruan J, Homer N, Marth G, Abecasis G, Durbin R. The sequence alignment/map format and SAMtools. Bioinformatics. 2009;25(16):2078-9.

27. Liao Y, Smyth GK, Shi W. featureCounts: an efficient general purpose program for assigning sequence reads to genomic features. Bioinformatics. 2014;30(7):923-30.

28. Love MI, Huber W, Anders S. Moderated estimation of fold change and dispersion for RNA-seq data with DESeq2. Genome Biol. 2014:15(12):550.

29. Zhou Y, Zhou B, Pache L, Chang M, Khodabakhshi AH, Tanaseichuk O, Benner C, Chanda SK. Metascape provides a biologist-oriented resource for the analysis of systems-level datasets. Nat Commun. 2019;10(1):1523.

30. Piñero J, Bravo À, Queralt-Rosinach N, Gutiérrez-Sacristán A, Deu-Pons J Centeno E, García-García J, Sanz F, Furlong LI. DisGeNET: a comprehensive platform integrating information on human disease-associated genes and variants. Nucleic Acids Res. 2017;45(D1):D833-9.

31. Bult CJ, Blake JA, Smith CL, Kadin JA, Richardson JE. The Mouse Genome Database G: Mouse Genome Database (MGD) 2019. Nucleic Acids Res. 2019:47(D1):D801-6.

32. Zhang P, McGrath B, Li S, Frank A, Zambito F, Reinert J, Gannon M, Ma K, McNaughton K, Cavener Douglas R. The PERK eukaryotic initiation factor $2 a$ kinase is required for the development of the skeletal system, postnatal growth, and the function and viability of the pancreas. Mol Cell Biol. 2002;22(11):3864-74.

33. Mortazavi A, Williams BA, McCue K, Schaeffer L, Wold B. Mapping and quantifying mammalian transcriptomes by RNA-SEq. Nat Methods. 2008:5(7):621-8.

34. Gu H, Zhu T, Li X, Chen Y, Wang L, Lv X, Yang W, Jia Y, Jiang Z, Qu L. A joint analysis strategy reveals genetic changes associated with artificial selection between egg-type and meat-type ducks. Anim Genet. 2020;51(6):890-8. 
35. Liu R, Liu W, Rong E, Lu L, Li H, Chen L, Zhao Y, Cao H, Liu W, Chen $C$, et al: Genomic analyses reveal the origin of domestic ducks and identify different genetic underpinnings of wild ducks. bioRxiv 2020:2020.2002.2003.933069.

36. Aguado E, Pascaretti-Grizon F, Goyenvalle E, Audran M, Chappard D. Bone mass and bone quality are altered by hypoactivity in the chicken. PLOS ONE. 2015;10(1):e0116763.

37. Jahnavi S, Poovazhagi V, Kanthimathi S, Gayathri V, Mohan V, Radha V. EIF2AK3 mutations in South Indian children with permanent neonatal diabetes mellitus associated with Wolcott-Rallison syndrome. Pediatr Diabetes. 2014;15(4):313-8.

38. Senée V, Vattem KM, Delépine M, Rainbow LA, Haton C, Lecoq A, Shaw NJ, Robert JJ, Rooman R, Diatloff-Zito C, et al. Wolcott-Rallison Syndrome: clinical, genetic, and functional study of EIF2AK3 mutations and suggestion of genetic heterogeneity. Diabetes. 2004;53(7):1876-83.

39. Reis AF, Kannengiesser C, Jennane F, Manna TD, Cheurfa N, Oudin C, Savoldelli RD, Oliveira C, Grandchamp B, Kok F, et al. Two novel mutations in the EIF2AK3 gene in children with Wolcott-Rallison syndrome. Pediatr Diabetes. 2011;12(3 Pt 1):187-91.

40. Harding HP, Zeng H, Zhang $Y$, Jungries $R$, Chung P, Plesken $H$, Sabatini DD, Ron D. Diabetes mellitus and exocrine pancreatic dysfunction in perk/- mice reveals a role for translational control in secretory cell survival. Molecular cell. 2001;7(6):1153-63.

41. Campagna L, McCracken KG, Lovette IJ. Gradual evolution towards flightlessness in steamer ducks*. Evolution. 2019;73(9):1916-26.

42. Burga A, Wang W, Ben-David E, Wolf PC, Ramey AM, Verdugo C, Lyons K, Parker PG, Kruglyak L. A genetic signature of the evolution of loss of flight in the Galapagos cormorant. Science. 2017;356(6341):eaal3345.

43. Williams M. Size and flight capability of Anas chathamica, an extinct duck from Chatham Island, New Zealand. Wildfowl. 2015;65:75.

44. Stunden CE, Bluhm CK, Cheng KM, Rajamahendran R. Factors affecting reproductive performance in captive mallard ducks. Theriogenology. 1999;52(3):435-46.

45. Dial TR, Heers AM, Tobalske BW. Ontogeny of aerodynamics in mallards: comparative performance and developmental implications. J Exp Biol. 2012;215(21):3693.
46. Chang W, Xie Q, Zheng A, Zhang S, Chen Z, Wang J, Liu G, Cai H. Effects of aflatoxins on growth performance and skeletal muscle of Cherry Valley meat male ducks. Animal Nutr. 2016;2(3):186-91.

47. Zhang YR, Zhang LS, Wang Z, Liu Y, Li FH, Yuan JM, Xia ZF. Effects of stocking density on growth performance, meat quality and tibia development of Pekin ducks. ANIM SCI J. 2018;89(6):925-30.

48. Kolbe EW, Tamm S, Hedtfeld S, Becker T, Tümmler B, Stanke F. CLCA4 variants determine the manifestation of the cystic fibrosis basic defect in the intestine. Eur J Hum Genetics. 2013;21(6):691-4.

49. Ritzka M, Stanke F, Jansen S, Gruber AD, Pusch L, Woelfl S, Veeze HJ, Halley DJ, Tümmler B. The CLCA gene locus as a modulator of the gastrointestinal basic defect in cystic fibrosis. Human Genetics. 2004;115(6):483-91.

50. Sanchez-Pulido L, Ponting CP. TMEM132: an ancient architecture of cohesin and immunoglobulin domains define a new family of neural adhesion molecules. Bioinformatics. 2018;34(5):721-4.

51. Li J, Zhao X, Xin Q, Shan S, Jiang B, Jin Y, Yuan H, Dai P, Xiao R, Zhang Q, et al. Whole-exome sequencing identifies a variant in TMEM132E causing autosomal-recessive nonsyndromic hearing loss DFNB99. Hum Mutat. 2015;36(1):98-105.

52. Lane JM, Liang J, Vlasac I, Anderson SG, Bechtold DA, Bowden J, Emsley R, Gill S, Little MA, Luik Al, et al. Genome-wide association analyses of sleep disturbance traits identify new loci and highlight shared genetics with neuropsychiatric and metabolic traits. Nat Genet. 2017;49(2):274-81.

53. Qanbari S, Pausch H, Jansen $S$, Somel M, Strom TM, Fries R, Nielsen R, Simianer $\mathrm{H}$. Classic selective sweeps revealed by massive sequencing in cattle. PLOS Genetics. 2014;10(2):e1004148.

\section{Publisher's Note}

Springer Nature remains neutral with regard to jurisdictional claims in published maps and institutional affiliations.
Ready to submit your research? Choose BMC and benefit from:

- fast, convenient online submission

- thorough peer review by experienced researchers in your field

- rapid publication on acceptance

- support for research data, including large and complex data types

- gold Open Access which fosters wider collaboration and increased citations

- maximum visibility for your research: over $100 \mathrm{M}$ website views per year

At BMC, research is always in progress.

Learn more biomedcentral.com/submissions 